\title{
Targeted Axillary Biopsy with Preoperative Ultrasound-Guided Tattooing for Suspicious Axillary Lymph Nodes in Patients with Early Breast Cancer
}

\author{
Jin Hyang Jung, Jeeyeon Lee, Wan Wook Kim, Ho Yong Park, Ji-Young Park ${ }^{1}$, Sang Woo Lee ${ }^{2}$, Won Hwa Kim ${ }^{3}$, \\ Hye Jung Kim \\ Department of Surgery, ${ }^{1}$ Department of Pathology, ${ }^{2}$ Department of Nuclear Medicine, ${ }^{3}$ Department of Radiology, School of Medicine, \\ Kyungpook National University, Kyungpook National University Chilgok Hospital, Daegu, Korea
}

Received September 14, 2020 Revised October 12, 2020

Accepted October 12, 2020

\begin{abstract}
Purpose: This study evaluated the efficiency of preoperative ultrasound (US)-guided tattooing of the axillary lymph nodes with activated charcoal and the correlation between sonographically suspicious nodes and final histologic results by node-to-node analysis. The concordance rate between the tattooed nodes and sentinel nodes was also determined.

Methods: US-guided tattooing of sonographically suspicious axillary nodes was performed preoperatively by an injection of activated charcoal. The identification of black pigment and the concordance between the sentinel and tattooed nodes was evaluated.

Results: Regarding node-to-node analysis, the false-negative rate of US-fine needle aspiration (FNA) was $43.3 \%$. The sensitivity and negative predictive values were $56.7 \%$ and $81.7 \%$, respectively. The specificity and positive predictive values were $100 \%$. The accuracy of US-FNA was $85.2 \%$. In the final pathology, 45/125 patients (36.0\%) had positive nodes, including two micrometastases. The false-negative rate of sentinel lymph node biopsy (SLNB) was $4.0 \%$, but there were no skip metastases. The sensitivity and specificity of SLNB were $95.6 \%$ and $100 \%$, respectively. The negative predictive value was $97.6 \%$, and the positive predictive value was $100 \%$. The accuracy of SLNB was $98.4 \%$. In 117 of 125 patients (93.6\%), there was concordance between the charcoal tattooed axillary lymph nodes and SLNs.

Conclusion: SLNB, in conjunction with US-guided tattooing of sonographically suspicious axillary lymph nodes, is a useful procedure to reduce the false-negative rate of SLNB and improve the accuracy of an intraoperative evaluation of axillary nodes in breast cancer patients. This paper proposes the concept of targeted axillary node biopsy with preoperative US-guided tattooing for the most accurate axillary staging in patients with breast cancer.
\end{abstract}

Keywords: Breast cancer, Lymph nodes, Axilla, Tattooing, Charcoal

\section{INTRODUCTION}

Accurate assessment of axillary lymph node (ALN) status is essential for staging and determining the optimal treat- ment in breast cancer. In clinically node-negative breast cancer, sentinel lymph node biopsy (SLNB) is the standard procedure for axillary staging, instead of complete axillary lymph node dissection (ALND).(1,2) Patients without SLN

Journal of Surgical Ultrasound is an Open Access Journal. All articles are distributed under the terms of the Creative Commons Attribution Non-Commercial License (http://creativecommons org/licenses/by-nc/4.0/) which permits unrestricted non-commercial use, distribution, and reproduction in any medium, provided the original work is properly cited. 
metastases do not need to undergo ALND because there is no survival benefit to this procedure. $(3,4)$ Previous guidelines recommend axillary dissection in all patients with metastatic findings on SLNB. Today, even in node-positive patients, the routine use of ALND is being questioned. The 2014 American Society of Clinical Oncology (ASCO) guidelines state that patients with one or two positive nodes undergoing breast conserving surgery and whole breast radiotherapy, who fulfill the Z0011 entry criteria, do not require routine ALND.(3) With changes in practice, the need for more accurate axillary staging is increasing.

The major concern with SLNB is the existence of a false negative rate of 5-10\%.(4-6) Skip metastasis of axillary nodes in breast cancer, which has an incidence of about 1.5$19.2 \%$, poses a challenge in relation to the safety of SLNB. $(7,8)$ In cases of skip metastasis, the results obtained via SLNB may be false negative. This potentially increasing false negative rate of SLNB is of concern when positive lymph nodes are not removed.

A number of recent studies have addressed preoperative axillary ultrasound (US) and US-guided fine needle aspiration (US-FNA) as alternative/additional staging procedures to improve the accuracy of SLNB. However, US-FNA resulted in highly variable false negative rates, particularly a lower nodal burden, thus compromising the use of the procedure in the clinical setting.(9-11) Moreover, the correlation between the ALNs identified on US and the SLN identified at surgery has not been fully evaluated. It is not known how often a lymph node visualized and biopsied on axillary US is one of the lymph nodes removed on SLNB. Which lymph node is biopsied under US guidance at presentation is dependent on sonographic visualization. The indication for biopsy is based on size and morphological features identified sonographically.(12) Lymph nodes removed at SLNB are identified by entirely different criteria with use of lymphatic mapping agents, such as radioisotopeand/or blue dye, injected into the breast to identify the draining lymph nodes. In the neoadjuvant setting, a procedure involving marking of the biopsied ALN with metal clips is being adopted. However, there are few reports regarding pre- operative marking in operable breast cancer with lower axillary nodal burden.

In the present study, our purpose was to evaluate the efficiency of preoperative US-guided tattooing of ALNs with activated charcoal and to correlate sonographically suspicious lymph nodes with final histologic results through node-to-node analysis. We also aimed todetermine the concordance rate between tattooed lymph nodes and SLNs detected by radioisotope and blue dye.Finally, we proposed the concept of targeted axillary node biopsy with preoperative US-guided tattooing for most accurate axillary staging in patients with breast cancer.

\section{METHODS}

Between January 2014 and February 2017, a total of 125 women with histologically confirmed primary operable breast cancer and one suspicious ALN on preoperative US were deemed eligible for the present study. Patients with multiple lymph node metastasis on preoperative imaging and those undergoing neoadjuvant therapy were excluded. All patients had undergone axillary US before SLNB. Detected lymph nodes were considered suspicious based on any one of the following characteristics: cortical thickness $\geq 2 \mathrm{~mm}$, eccentric cortical thickening, loss of fatty hilum, diameter of short axis $>10 \mathrm{~mm}$, round shape (L/S ratio $<1.5)$.(13-15) In all, 93 patients underwent US-FNA for suspicious nodes prior to surgery.

US-guided tattooing was performed on all lymph nodes with suspicious US characteristics. The procedure was performed preoperatively by injection of $1-3 \mathrm{~mL}$ of Charcotrace $^{\mathrm{TM}}$ (activated charcoal $120 \mathrm{mg}$ and sodium chloride in water for injections to $3 \mathrm{~mL}$, Phebra, Lane Cove West, NSW, Australia) into the cortex of the lymph node and the adjacent soft tissue after local anesthesia (Fig. 1). For all SLNB, mapping agents, including radioisotope (technetium-99m phytate) and/or blue dye, were injected before or at the time of surgery. During surgery, the axilla was inspected to determine whether black charcoal tattoo was visible in the node or soft tissue. Lymph nodes identi- 

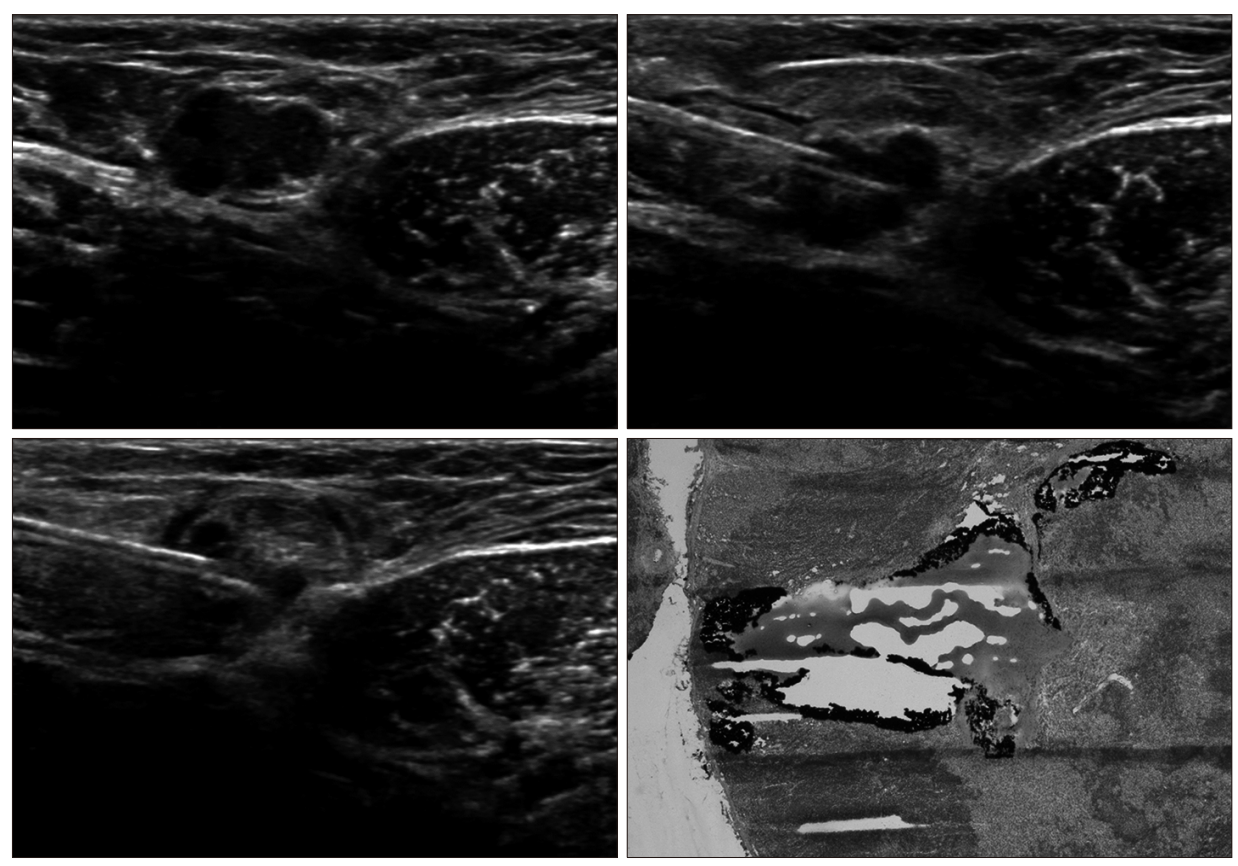

Fig. 1. Ultrasound-guided localization using activated charcoal for the suspicious axillary lymph node.
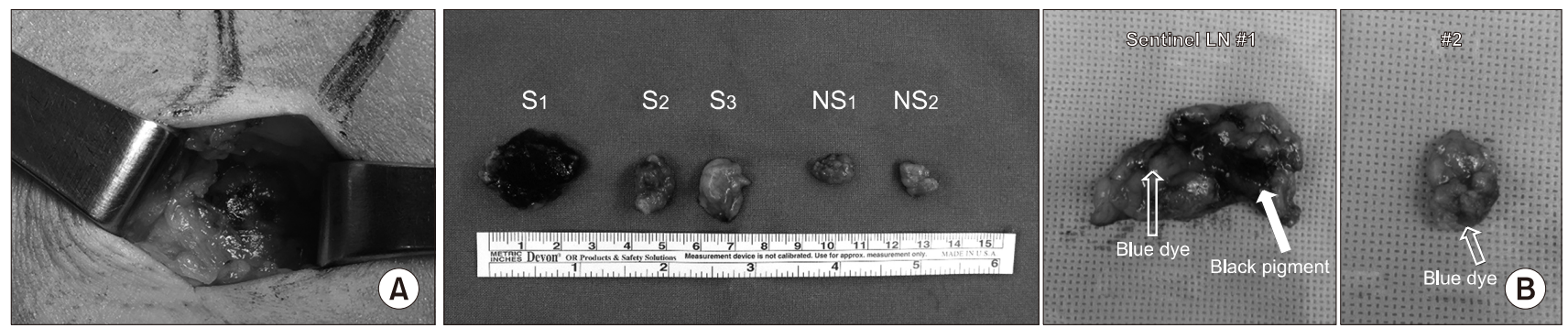

Fig. 2. Identification of black pigment (A) and evaluation of concordance between sentinel and tattooed node (B).

fied by a gamma probe or those containing blue dye were regarded as sentinel nodes. All SLNs and tattooed lymph nodes were removed, and the concordance was determined (Fig. 2). Palpable nodes without radioisotope uptake and blue dye were also removed and labeled as non-sentinel nodes.

Each removed lymph node was examined using frozen sections. If a frozen section was positive for metastasis, ALND was performed according to 2014 ASCO guidelines.(3) ALND was omitted in patients with one or two positive nodes undergoing breast-conserving surgery and whole-breast radiotherapy. All tattooed lymph nodes were compared with final histologic results through node-to-node analysis. Each lymph node was finally classified as negative or positive for metastasis. Lymph nodes with micrometastasis were classified as positive for metastasis, and nodes with isolated tumor cells were classified as negative.

The false negative rate was calculated as the number of false negative events divided by the total number of pathological positive nodes. Statistical analysis was performed using SPSS software (ver. 24.0; IBM Corp., Chicago, IL, USA). Confidence intervals (CIs) for false negative rates were calculated using exact Clopper-Pearson confidence limits for the binomial proportion. This study was approved by the Institutional Review Board of Kyungpook National University Chilgok Hospital (KNUCH 2017-02013), and written informed consent was obtained from each patient before study enrollment. 


\section{RESULTS}

Clinicopathologic and treatment details of the 125 enrolled patients were described in Table 1. SLNB using both isotope and/or blue dye was successful in all patients. During axillary procedure, all of the tattooed lymph nodes were easily identified by visual inspection and removed in

Table 1. Clinicopathologic Characteristics of Patients

\begin{tabular}{|c|c|}
\hline Characteristics & No. $(\%)$ of patients $(\mathrm{n}=125)$ \\
\hline Mean age, years & 49.7 , range $31-75$ \\
\hline Mean tumor size of IDC, $\mathrm{cm}$ & 2.1 , range $0.1-6.0$ \\
\hline \multicolumn{2}{|l|}{ T stage } \\
\hline T0 & 4 \\
\hline $\mathrm{T} 1$ & 63 \\
\hline $\mathrm{T} 2$ & 56 \\
\hline T3 & 2 \\
\hline \multicolumn{2}{|l|}{ N stage } \\
\hline NO & 80 \\
\hline N1 & 38 \\
\hline $\mathrm{N} 2$ & 4 \\
\hline N3 & 3 \\
\hline \multicolumn{2}{|l|}{ Tumor receptor subtype } \\
\hline HR+/HER2- & 60 \\
\hline $\mathrm{HR}+/ \mathrm{HER} 2+$ & 17 \\
\hline HR-/HER2 + & 20 \\
\hline HR-/HER2- & 28 \\
\hline \multicolumn{2}{|l|}{ Type of breast surgery } \\
\hline BCT & 82 \\
\hline Mastectomy & 43 \\
\hline
\end{tabular}

IDC = invasive ductal carcinoma; HR = hormone receptor; HER2 $=$ human epidermal growth factor receptor 2 ; $\mathrm{BCT}=$ breast conservation therapy. all patients. No significant adverse reactions occurred with charcoal tattooing. A mean of 1.86 (range, 1 to 6) SLN were removed, with 1 SLN in 52 patients, 2 SLNs in 48 patients, and $\geq 3$ SLNs in 25 patients. The mean number of non-sentinel nodes removed was 1.44 (range, 0 to 5). Frozen section examination of SLN revealed that 43 cases (34.4\%) had positive results. Of them, 22 cases were performed ALND and the other 21 cases with one or two positive nodes were omitted ALND according to 2014 ASCO guideline.(3) Two patients had negative finding in frozen section, but final pathology showed micrometastasis in that node.

From the tattooed node-based analysis, of the 125 nodes considered as suspicious in US, 43 (34.4\%) were positive for metastasis and 82 (65.6\%) were negative. 94 (75.2\%) patients underwent US-FNA of ALN with suspicious imaging finding. The result was positive in 17 cases (18.1\%), negative in 71 cases (75.5\%). Six cases (6.4\%) showed the results of atypia of undetermined significance. These cases in which FNA findings were indeterminate were analyzed separately by comparing them to final surgical pathologic findings. Regarding the node-to-node analysis, of the US-FNA results, 17 (19.3\%) of 88 were true-positive; 58 (65.9\%), true-negative; 13 (14.8\%), false-negative; zero (0\%), false-positive (Fig. 3). False-negative rate of US-FNA was $43.3 \%$ (95\% CI: 25.6 to 61.1). Sensitivity of US-FNA for sonographically suspicious node was $56.7 \%$ (95\% CI: 38.9 to 74.4). Negative predictive value was $81.7 \%$ (95\% CI: 72.7 to 90.1). But, specificity and positive predictive value were

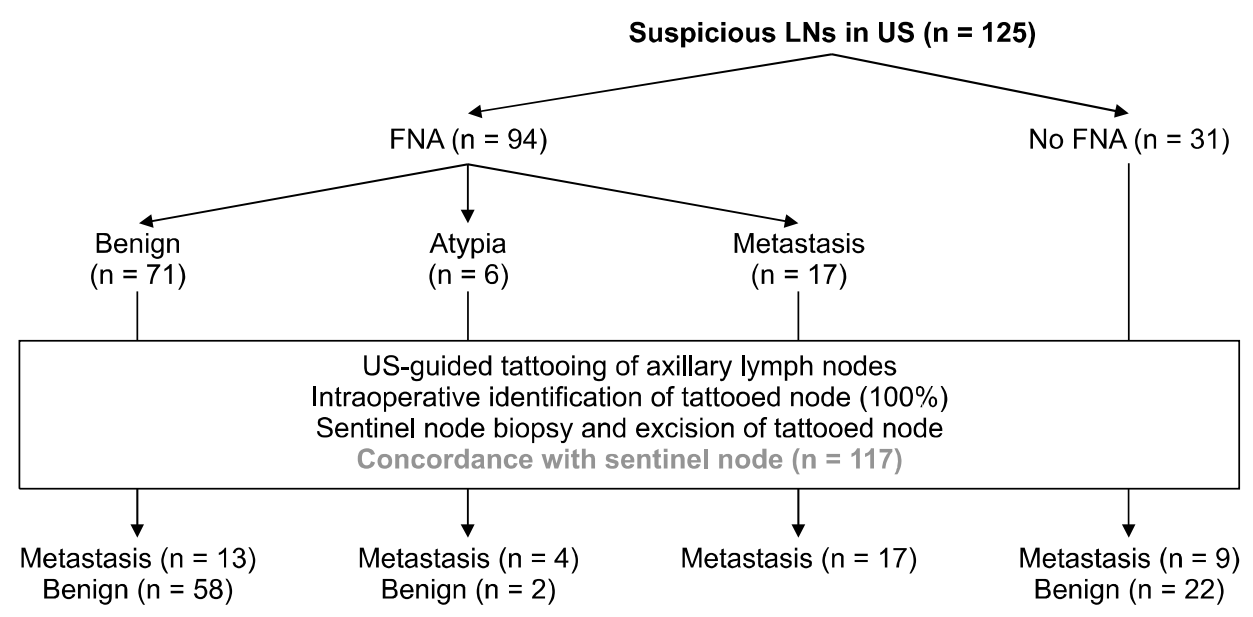

Fig. 3. Schematic of patients. $\mathrm{LN}=$ lymph node; FNA = fine needle aspiration; US $=$ ultrasound. 
Table 2. Patients with Discordance between the Tattooed Nodes and Sentinel Nodes

\begin{tabular}{|c|c|c|c|c|c|c|c|c|c|c|c|c|}
\hline \multirow{2}{*}{$\begin{array}{l}\text { Case } \\
\text { No. }\end{array}$} & \multirow{2}{*}{ Sex/age } & \multirow{2}{*}{ Breast OP } & \multirow{2}{*}{$\begin{array}{c}\text { Axilla } \\
\text { OP }\end{array}$} & \multirow{2}{*}{$\begin{array}{c}\text { USG LN } \\
\text { size }(\mathrm{mm})\end{array}$} & \multirow{2}{*}{ FNA result } & \multirow{2}{*}{$\begin{array}{l}\text { No of } \\
\text { SLN }\end{array}$} & \multicolumn{2}{|c|}{ Frozen $\mathrm{Bx}$} & \multicolumn{2}{|c|}{ Final Bx } & \multirow{2}{*}{$\begin{array}{c}\text { No of } \\
\text { metastatic } \\
\text { LN }\end{array}$} & \multirow{2}{*}{$\begin{array}{c}\text { Skip } \\
\text { metastasis }\end{array}$} \\
\hline & & & & & & & SLN & Tattooed node & SLN & Tattooed node & & \\
\hline 1 & $\mathrm{~F} / 55$ & Mastectomy & SLNB & 20 & Benign & 2 & Negative & Negative & Negative & Negative & 0 & - \\
\hline 2 & $\mathrm{~F} / 61$ & BCS & $\mathrm{AD}$ & 10 & Benign & 1 & Positive & Positive & Positive & Positive & 2 & No \\
\hline 3 & $\mathrm{~F} / 46$ & BCS & SLNB & 20 & Not done & 1 & Negative & Negative & Negative & Negative & 0 & - \\
\hline 4 & $\mathrm{~F} / 41$ & BCS & $\mathrm{AD}$ & 7 & Malignancy & 1 & Positive & Positive & Positive & Positive & 4 & No \\
\hline 5 & $F / 36$ & BCS & SLNB & 11 & Benign & 1 & Negative & Negative & Negative & Negative & 0 & - \\
\hline 6 & $\mathrm{~F} / 48$ & Mastectomy & SLNB & 7 & Benign & 2 & Negative & Negative & Negative & Negative & 0 & - \\
\hline 7 & $\mathrm{~F} / 75$ & Mastectomy & AS & 10 & Benign & 3 & Negative & Negative & Negative & Negative & 0 & - \\
\hline 8 & $\mathrm{~F} / 43$ & BCS & $\mathrm{AD}$ & 10 & Malignancy & 2 & Positive & Positive & Positive & Positive & 3 & No \\
\hline
\end{tabular}

$\mathrm{No}=$ number, $\mathrm{OP}=$ operation, $\mathrm{BCS}=$ breast conserving surgery, $\mathrm{SLNB}=$ sentinel lymph node biopsy, $\mathrm{AD}=$ axillary dissection, $\mathrm{AS}=$ axillary sampling, $\mathrm{FNA}=$ fine needle aspiration, $\mathrm{Bx}=$ biopsy.

$100 \%$. Accuracy of US-FNA for suspicious axillary nodes was $85.2 \%$ (95\% CI: 77.8 to 92.6). Follow-up of the 6 cases with an indeterminate result in US-FNA revealed that four were lymph node positive as lymph node negative $(n=2)$. And all these 6 cases matched sentinel nodes.

In final pathology, 45 patients (36.0\%) had positive node including two micrometastases. False-negative rate of SLNB was $4.4 \%$ (95\% CI: 1.6 to 10.5$)$, but there was no skip metastasis. Sensitivity and specificity of SLNB were $95.6 \%$ (95\% CI: 89.5 to 101.6) and 100\% respectively. Negative predictive value was $97.6 \%$ (95\% CI: 94.2 to 100.9 ), and positive predictive value was $100 \%$. Accuracy of SLNB was 98.4\% (95\% CI: 96.2 to 100.6$)$.

In 117 of 125 patients (93.6\%), there was concordance between charcoal tattooed ALNs and SLNs. Eight patients (6.4\%) had discordance between the tattooed nodes and SLNs. Among the patients with discordance, three patients had metastasis in the tattooed nodes, but also had metastasis in one or two SLNs. The other five patients had no metastasis in either tattooed or sentinel nodes (Table 2).

\section{DISCUSSION}

With changing practice, routine ALND could be omitted in the patients who fulfil Z0011 entry criteria.(3) Therefore the needs for more accurate axillary staging are increasing. SLNB has been proven to be a valid method of assessing
ALN status in early breast cancer. However, the major concern with SLNB is the existence of false negative rate of 5 to $10 \%$.(4-6) This potentially increasing false negative rate of SLNB was concerned if the positive lymph node was not excised. And also, the correlation between the ALN identified on preoperative axillary US and the SLN identified at surgery has not been fully evaluated.

In present study, black-tattooed ALNs could be easily detected in all patients during SLNB, fulfilling the technical feasibility of node tattooing without adverse reactions. Intraoperatively, the black pigment from tattooing was well distinguished from blue-stained SLN. Other techniques have been introduced to mark positive lymph nodes, such as metallic markers and radioactive seeds. $(2,16,17)$ Our nodal tattooing technique with activated charcoal is more simple, convenient and cost-effective than other techniques. It required no additional imaging to ascertain the location of marker at the time of definitive surgery. Furthermore, the tattooing did not interfere with a standard SLNB and allowed for correlation to preoperative-biopsied lymph nodes.

Multiple studies reported the efficacy of US-FNA for preoperative axillary staging in early breast cancer. According to a systematic review reported by Yu et al.(18) the pooled analysis has shown the sensitivity of US-FNA ranged from 40 to $90 \%$ and the specificity ranged from 93 to $100 \%$. False-negative rate was approximately $34 \%$. However, 
those studies could not precisely determine false-negative rate because node-to-node analysis was unavailable. One of the major strength of this study is the direct correlation between the FNA results of suspicious lymph node on US and final pathologic results through performing node-tonode match with US-guided tattooing. The sensitivity and specificity of US-FNA for sonographically suspicious axillary node were $56.7 \%$ and $100 \%$, respectively. False-negative rate was $43.3 \%$. The sensitivity and specificity are comparable but false-negative rate is slightly higher than the range of rate (29 to $40 \%$ ) reported by meta-analysis.(18) This results of preoperative US-FNA, only $19.3 \%$ of cases resulted truly positive, with $14.8 \%$ being false negative, demonstrated the unsatisfying accuracy of the procedure. In this study, of the 6ALNs with atypia on FNA, 4 ALNs (66.7\%) had metastasis in the final pathology. It is questionable whether atypical cell on FNA should be considered positive or negative for metastasis. For that reason, we suggest SLNB with charcoal tattooing in the patients who have ALNs with atypia on cytology. This procedure can be reduced repeated FNA or unnecessary ALNDs.

According to ASCO guidelines for SLNB, six of the trials to date have reported on performance aspects of SLNB. False-negative rate of SLNB reported in the six studies ranged from approximately $4.6 \%$ to $16.7 \%$.(3) Three studies reported negative predictive value ranging from $90.1 \%$ to $96.1 \%$. Four studies reported overall accuracy of SNB results ranging from $93 \%$ to $97.6 \%$. In present study, negative predictive value and accuracy of SLNB was $97.6 \%$ and $98.4 \%$, respectively. False-negative rate was $4.4 \%$, but there was no skip metastasis. The failure to identify micrometastases in SLN with frozen biopsy was the cause of these false-negative results.

The correlation between the ALNs identified on US and the SLN identified at surgery has not been fully evaluated. Previous studies have shown that an overlap exists between the SLNs and the suspicious lymph nodes. $(19,20)$ Nathanson (21) reported a correlation between ALNs identified by percutaneous biopsy and ALNs resected at the time of SLNB of $78 \%$. In the present study, most suspicious lymph nodes
(117/125, 93.6\%) were indeed SLNs. The reason that most of the suspicious ALNs on US were SLNs with a higher concordance rate is unclear. While suspicious lymph nodes on US are identified by their size and morphological features, SLNs are hypothetical lymph nodes or groups of nodes draining a cancer. US and surgical SLN concordance are enhanced when the node is morphologically abnormal. This study, which was not restricted to patients with positive nodes, suggests that in the majority of cases, the nodes resected at SLNB include the node that is identified on US. However, the concordance is not 100 percent and at times, suspicious nodes identified by US is not one of the SLNs. This discordance is concerned if the positive lymph node is not removed. Marking the biopsied axillary node with percutaneous tattooing is helpful to identify previously biopsied node. Selective removal of sonographically suspicious $\mathrm{LN}$ as well as SLNs may improve the ability to accurately stage the axilla. Thus, SLNB was easier to conduct, and the possibility of missing a positive lymph node was reduced.

This study was limited to the patients with only one suspicious lymph node on preoperative axillary US. This was because two or more suspicious lymph nodes on US may be expected to be higher burden nodal disease. The patients with higher burden of axillary disease would not fit criteria forinclusion in the Z0011 trial, thereby undergoing ALND. There are other limitations. The determination of suspicious ALNs on US was subjective and potential variability among radiologists can occur. US-FNA is an operator-dependent procedure for obtaining the desired tissue. And of the patients with positive results in frozen section examination of SLN, 21 (49\%) cases with one or two positive nodes were omitted ALND. Therefor we cannot entirely rule out the possibility of skip metastasis for the patients who only underwent SLNB. However, we believe that this limitation is inevitable because routine ALND is not currently recommended in breast cancer patients. Also, we think that our method of surgical management for ALNs may not have much effect on our results. This study has single cohort design and small sample size. Thus, to apply with the results in clinical setting, much larger series or 
randomized control studies will be needed.

\section{CONCLUSION}

In our preliminary study, SLNB in conjunction with US-guided tattooing of sonographically suspicious axillary lymph node is a useful procedure to reduce the false-negative rate of SLNB and to improve the accuracy of intraoperative evaluation of the axillary nodes in patients with early breast cancer. It is expected that preoperative US-FNA can be replaced by US-guided nodal tattooing. So, we proposed the concept of targeted axillary node biopsy with preoperative US-guided tattooing for most accurate axillary staging in patients with early breast cancer.

\section{CONFLICT OF INTEREST}

The authors declare that they have no conflict of interest.

\section{REFERENCES}

1. Naik AM, Fey J, Gemignani M, Heerdt A, Montgomery L, Petrek J, et al. The risk of axillary relapse after sentinel lymph node biopsy for breast cancer is comparable with that of axillary lymph node dissection: a follow-up study of 4008 procedures. Ann Surg 2004;240:462-8; discussion 468-71.

2. Caudle AS, Cupp JA, Kuerer HM. Management of axillary disease. Surg Oncol Clin N Am 201423:473-86.

3. Lyman GH, Temin S, Edge SB, Newman LA, Turner RR, Weaver DL, et al. Sentinel lymph node biopsy for patients with early-stage breast cancer: American Society of Clinical Oncology clinical practice guideline update. J Clin Oncol 2014;32: 1365-83.

4. Krag DN, Anderson SJ, Julian TB, Brown AM, Harlow SP, Ashikaga T, et al. Technical outcomes of sentinel-lymph-node resection and conventional axillary-lymph-node dissection in patients with clinically node-negative breast cancer: results from the NSABP B-32 randomised phase III trial. Lancet Oncol 2007:8:881-8.

5. White RL Jr, Wilke LG. Update on the NSABP and ACOSOG breast cancer sentinel nodetrials. Am Surg 2004;70:420-4.

6. Goyal A, Newcombe RG, Chhabra A, Mansel RE ALMANAC Trialists Group. Factors affecting failed localisation and false-negative rates of sentinel node biopsy in breast cancer--results of the ALMANAC validation phase. Breast Cancer Res Treat 2006;99:203-8.

7. Wang H, Mao XY, Zhao TT, Zheng XY, Jin F, Li JG. Study on the skip metastasis of axillary lymph nodes in breast cancer and their relation with Gli1 expression. Tumour Biol 2012;33: 1943-50.

8. Sun J, Yin J, Ning L, Liu J, Liu H, Gu L, et al. Clinicopathological characteristics of breast cancers with axillary skip metastases. J Invest Surg 2012;25:33-6.

9. van Rijk MC, Teertstra HJ, Peterse JL, Nieweg OE, Olmos RA, Hoefnagel CA, et al. Ultrasonography and fine-needle aspiration cytology in the preoperative evaluation of melanoma patients eligible for sentinel node biopsy. Ann Surg Oncol 2006;13:1511-6.

10. Krishnamurthy S, Sneige N, Bedi DG, Edieken BS, Fornage BD, Kuerer HM, et al. Role of ultrasound-guided fine-needle aspiration of indeterminate and suspicious axillary lymph nodes in the initial staging of breast carcinoma. Cancer 200295:982-8.

11. Swinson C, Ravichandran D, Nayagam M, Allen S. Ultrasound and fine needle aspiration cytology of the axilla in the pre-operative identification of axillary nodal involvement in breast cancer. Eur J Surg Oncol 2009;35:1152-7.

12. Boughey JC, Ballman KV, Hunt KK, McCall LM, Mittendorf EA, Ahrendt GM, et al. Axillary ultrasound after neoadjuvant chemotherapy and its impact on sentinel lymph node surgery: results from the American College of Surgeons Oncology Group Z1071 trial (Alliance). J Clin Oncol 2015;33:3386-93.

13. Hyun SJ, Kim EK, Moon HJ, Yoon JH, Kim MJ. Preoperative axillary lymph node evaluation in breast cancer patients by breast magnetic resonance imaging (MRI): can breast MRI exclude advanced nodal disease? Eur Radiol 2016;26:3865-73.

14. Park SH, Kim EK, Park BW, Kim SI, Moon HJ, Kim MJ. False negative results in axillary lymph nodes by ultrasonography and ultrasonography-guided fine-needle aspiration in patients with invasive ductal carcinoma. Ultraschall Med 2013; 34:559-67.

15. Cho N, Moon WK, Han W, Park IA, Cho J, Noh DY. Preoperative sonographic classification of axillary lymph nodes in patients with breast cancer: node-to-node correlation with surgical histology and sentinel node biopsy results. AJR Am J Roentgenol 2009;193:1731-7.

16. Donker M, Straver ME, Wesseling J, Loo CE, Schot M, Drukker $\mathrm{CA}$, et al. Marking axillary lymph nodes with radioactive iodine seeds for axillary staging after neoadjuvant systemic treatment in breast cancer patients: the MARI procedure. Ann Surg 2015;261:378-82

17. Caudle AS, Yang WT, Krishnamurthy S, Mittendorf EA, Black DM, Gilcrease MZ, et al. Improved axillary evaluation following neoadjuvant therapy for patients with node-positive breast cancer using selective evaluation of clipped nodes: implementation of targeted axillary dissection. J Clin Oncol 2016;34:1072-8

18. Yu YH, Mo QG, Zhu X, Gao LQ, Liang C, Huang Z, et al. Axillary fine needle aspiration cytology is a sensitive and highly specific technique for the detection of axillary lymph node metastasis: a meta-analysis and systematic review. Cytopathology 2016;27: 59-69.

19. Cserni G. Estimating the overlap between sentinel lymph nodes and axillary node samples in breast cancer. Pathol Oncol Res 1999;5:129-33.

20. Macmillan RD, Barbera D, Hadjiminas DJ, Rampaul RS, Lee 
$\mathrm{AH}$, Pinder SE, et al. Sentinel node biopsy for breast cancer may have little to offer four-node-samplers. results of a prospective comparison study. Eur J Cancer 2001;37:1076-80.
21. Nathanson SD. Preclinical models of regional lymph node tumor metastasis. Cancer Treat Res 2007;135:129-56. 